\title{
HER2 Status and Its Heterogeneity in Gastric Carcinoma of Vietnamese Patient
}

Dang Anh Thu Phan

Vu Thien Nguyen . Thi Ngoc Ha Hua

Quoc Dat Ngo

Thi Phuong Thao Doan

Sao Trung Nguyen · Anh Tu Thai ${ }^{1}$

Van Thanh Nguyen ${ }^{1}$

Department of Pathology, University of Medicine and Pharmacy Ho Chi Minh City,

Ho Chi Minh City; 'Department of Pathology,

Ho Chi Minh City Oncology Hospital, Ho Chi Minh

City, Vietnam

Received: February 22, 2017

Revised: April 14, 2017

Accepted: April 24, 2017

\section{Corresponding Author}

Dang Anh Thu Phan, MD, PhD

Department of Pathology, University of Medicine and Pharmacy Ho Chi Minh City, 217 Hong Bang Street, Ward 11, District 5, Ho Chi Minh City, Vietnam

Tel: +84-8-38558411

Fax: +84-8-38552304

E-mail: phandanganhthu@yahoo.com
Background: Human epidermal growth factor receptor 2 (HER2) is related to the pathogenesis and poor outcome of numerous types of carcinomas, including gastric carcinoma. Gastric cancer patients with HER2 positivity have become potential candidates for targeted therapy with trastuzumab. Methods: We investigated 208 gastric cancer specimens using immunohistochemistry (IHC), fluorescence in situ hybridization and dual in situ hybridization (ISH). We also investigated the concordance between IHC and ISH. The correlation between HER2 status and various clinicopathological findings was also investigated. Results: In total, $15.9 \%$ (33/208) and $24.5 \%$ (51/208) of gastric cancers showed HER2 gene amplification and protein overexpression, respectively. A high level of concordance between ISH and IHC analyses $(91.3 \%, \kappa=0.76)$ was found. A significant correlation between HER2 status and intestinal-type $(p<.05)$ and differentiated carcinomas $(p<.05)$ was also noted. The HER2 heterogeneity was high in gastric cancers; we found $68.8 \%$ phenotypic heterogeneity and $57.6 \%$ genotypic heterogeneity. Heterogeneity in HER2 protein expression and gene amplification showed a close association with diffuse histologic type and IHC 2+. Conclusions: HER2 protein overexpression and gene amplification were detected in $24.5 \%$ and $15.9 \%$ of gastric cancer specimens, respectively. Intestinal-type showed a higher level of HER2 protein overexpression and gene amplification than diffuse type. HER2 status also showed a significant relationship with well- and moderately-differentiated carcinomas. The ratio of phenotypic and genotypic heterogeneity of HER2 was high in gastric carcinomas and was associated with HER2 IHC 2+ and diffuse histologic type.

Key Words: Protein HER2; Stomach neoplasms; Protein overexpression; Immunohistochemistry; Gene amplification; Fluorescence in situ hybridization; Dual-color silver in situ hybridization
According to global cancer statistics from 2012 (GLOBOCAN 2012), gastric cancer remains the fourth most frequent cause of cancer-related mortality in developing countries. ${ }^{1}$ In Vietnam, the incidence of gastric cancer has been increasing in recent years and poor outcome was due to delayed diagnosis and advanced stage.

Conventional chemotherapy has not been very beneficial for gastric cancer. Targeted therapeutics, especially human epidermal growth factor receptor 2 (HER2) inhibitors, have recently shown more advantages in treating late-stage gastric cancer. ${ }^{2}$ The Trastuzumab for Gastric Cancer trial has proven the efficacy of humanized monoclonal antibody against HER2 protein, so-called trastuzumab, in the treatment of advanced gastric cancer or gastroesophageal junction cancer with HER2 positivity. $^{3}$

HER2 is a proto-oncogene encoded by ERBB2 on chromosome 17, also a member of the HER family. The intracellular domain of
HER2, a membrane-bound receptor tyrosine kinase, is responsible for extracellular signal transmission to initiate cellular signaling pathways such as mitogen-activated protein kinase, phosphoinositide 3-kinase, phospholipase $\mathrm{C}$, and protein kinase $\mathrm{C}$, leading to cell proliferation, cell survival, angiogenesis, migration, and invasion. ${ }^{4}$ HER2-positive status (protein overexpression and/or gene amplification) has been identified in several carcinomas, especially in breast cancer, where it has a frequency of $20 \%-25 \%$, suggesting its prognostic and predictive value in this carcinoma. ${ }^{5}$ On the other hand, in gastric cancer, the reported level of HER2 overexpression and amplification varies widely from $7 \%-27 \%,{ }^{6-10}$ and the prognostic value of HER 2 status is not commonly accepted.

This study aimed to detect HER2 status including protein expression and gene amplification, as well as investigate HER2 heterogeneity and the concordance between two methods (immunohistochemistry [IHC] and in situ hybridization). We also 
investigated the relationship between HER2 status and clinicopathological findings.

\section{MATERIALS AND METHODS}

\section{Patients and tissue specimens}

For this study, we retrospectively enrolled 208 gastric cancer patients who underwent curative gastrectomy at the University Medical Center, Ho Chi Minh City, Vietnam, from January 2012 to June 2015. No patients had received preoperative radiation or chemotherapy. All surgical specimens were promptly fixed in $10 \%$ buffered formalin, no longer than 30 minutes after resection, and for a fixing time of 8-48 hours. Clinicopathological features were recorded including age, sex, histologic type by Lauren, tumor differentiation, depth of invasion, lymph node status, and lymphovascular invasion.

\section{IHC staining}

We performed IHC staining for HER2 on the automated slide stainer (BenchMark XT), using monoclonal antibody 4B5 (Ventana Medical System, Tucson, AZ, USA) following the manufacturer's procedures. We incubated 5 - $\mu \mathrm{m}$ slices after antigen retrieval ( $10 \mathrm{mM}$ citrate buffer at $98^{\circ} \mathrm{C}$ for 40 minutes) with $4 \mathrm{~B} 5$ for 30 minutes. Subsequently 3,3'-diaminobenzidine was added for visualization. The sections were stained with hematoxylin for counterstaining and then cover-slipped. Negative controls (omission of primary antibody) and positive controls (gastric cancer tissue with known IHC HER2 3+) were included in each staining run.

\section{Evaluation of $\mathrm{IHC}$ results}

Slides were scored by a pathologist following the scoring system of Hofmann et al. ${ }^{6}$ The generally accepted cut-off definition for HER 2 membranous positivity is $10 \%$ of the tumor cells. A score of 0 was defined as no reactivity or membrane staining in $<10 \%$ of the tumor cells. Faint membrane staining was defined as a score of $1+$. Weak-to-moderate complete membrane staining was defined as a score of $2+$, and moderate-to-strong complete membrane staining was defined as a score of $3+$. Scores of $2+$ and $3+$ were defined as HER2 protein positive/over-expression. Phenotypic heterogeneity was defined as the proportion of tumor cells showing membranous staining in more than $10 \%$, but less than $50 \%$ of tumor cells.

\section{In situ hybridization}

We performed in-situ hybridization (ISH) for 208 cases of gastric cancer using dual-color silver in situ hybridization (SISH) (28 cases randomly selected) and fluorescence in situ hybridization (FISH) (the remaining 180 cases).

\section{Fluorescence in situ hybridization}

HER2 gene amplification was detected by FISH using a Pathvision HER2 DNA probe kit (Vysis Inc., Downers Grove, IL, USA) according to the manufacturer's instructions. We heated hybridization buffer, DNA probe, and purified water to $73^{\circ} \mathrm{C}$ for 5 minutes. Slides were dipped in a denaturing bath (70\% formamide $2 \times$ saline-sodium citrate [SSC]) for 5 minutes at $73^{\circ} \mathrm{C}$, then dehydrated in increasing ethanol concentrations, and then covered with the probe mixture. We incubated all slides for 30 minutes at $42^{\circ} \mathrm{C}$, then washed with $0.4 \times \mathrm{SSC} / 0.3 \%$ $\mathrm{NP}-40$ for 2 minutes, air-dried in darkness, counterstained with 4',6-diamidino-2-phenylindole (DAPI), and lastly covered with a cover-slip. The signals were visualized under fluorescence microscope (BX51, Olympus, Tokyo, Japan) and the images were captured and saved with Spectral Imaging software (Applied Spectral Imaging Inc., Carlsbad, CA, USA). HER2 gene expression is represented by discrete red signals or signal clusters and $\mathrm{Chr} 17$ as green signals.

\section{Dual-color SISH}

We performed automated SISH on the Ventana Benchmark XT platform (Ventana Medical Systems Inc.) following the manufacturer's protocols and using the INFORM HER2 DNA and Chromosome 17 probes. HER2 is identified by a dinitrophenyl (DNP) labeled probe and visualized utilizing ultraView SISH DNP Detection Kit. The Chr17 centromere is labeled with a digoxigenin (DIG) probe and detected using ultraView Red ISH DIG Detection Kit (Ventana Medical Systems Inc.). The signals were visualized under light microscopy (BX50, Olympus) in which HER2 expression appeared as discrete black signals (SISH) and Chr17 as red signals. The mean HER2/CEP17 ratio was calculated by counting signals in 20 tumor cells under high-power field $(\times 600)$.

\section{Evaluation of FISH and dual SISH results}

HER2 gene status was based on the ratio of HER2 gene copies and $\mathrm{Chr} 17$ copies in nuclei of tumor cells. HER2 gene status was defined as non-amplified if the HER2/Chr17 ratio was under 2.0 or amplified if the HER2/Chr17 ratio was 2.0 or more. If the HER2/CEP17 ratio was 1.8 or more but less than 2.0, 20 more tumor cells were investigated to determine the HER2 gene status. HER2 amplification was further sub-classified into low-level amplification (HER2/CEP17 ratio 2-5) and high-level amplification 


\section{(HER2/CEP17 ratio $\geq 5)$.}

Polysomy of chromosome 17 was set as more than 3 CEP17 signals per nuclei. Genotypic heterogeneity was defined by the existence of more than $5 \%$ but less than $50 \%$ of tumor cells with a HER2/CEP17 ratio higher than 2.0.

\section{Statistical analysis}

We used SPSS ver. 15.0 statistical software program (SPSS Inc., Chicago, IL, USA) for statistical analyses. The chi-square tests (Kappa chi-square test or chi-square test) were performed to analyze correlations between HER2 status and clinicopathological features. p-values below .05 indicate statistically significant results.

\section{RESULTS}

\section{Clinicopathological characteristics of patients}

Patient age ranged widely from 30 to 81 years (median, 58.2 years). There was a higher number of male patients $(n=139)$ than female patients $(n=69)$, with a male/female ratio of 2:1. Clinicopathological features are noted in Table 1.

\section{Overexpression of HER2}

HER2 protein expression in 208 gastric tissue samples was investigated by IHC staining (Fig. 1). Of the 208 gastric carcinoma

Table 1. Clinical and pathologic features of 208 cases of gastric cancer

\begin{tabular}{lc}
\hline Parameter & No. (\%) \\
\hline Sex & $139(66.8)$ \\
Male & $69(33.2)$ \\
Female & \\
Age & $30-82$ \\
Range & $58.2 \pm 11.6$ \\
Mean \pm standard deviation & \\
Histologic type by Lauren & $78(37.5)$ \\
Intestinal type & $109(52.4)$ \\
Diffuse type & $21(10.1)$ \\
Mixed & \\
Tumor differentiation & $77(37.0)$ \\
Differentiated & $131(63.0)$ \\
Undifferentiated & \\
Depth of invasion (pT stage) & $12(5.8)$ \\
T1 & $36(17.3)$ \\
T2 & $10(4.8)$ \\
T3 & $150(72.1)$ \\
\hline T4 & \\
Lymph node status (pN stage) & $87(41.8)$ \\
N0 & $121(58.2)$ \\
N1, 2, 3 & $57(27.4)$ \\
\hline Lymphovascular invasion &
\end{tabular}

tissue samples, $148(71.2 \%)$ were scored as $0,9(4.3 \%)$ scored as $1+, 23(11.1 \%)$ scored as $2+$, and $28(13.4 \%)$ scored as $3+$. In total, $24.5 \%$ were positive $(51 / 208)$.

\section{HER2 amplification}

The amplification rate of the HER2 gene was 15.9\% (33/208) in all gastric carcinoma tissue samples, with 29 cases detected by FISH and four cases detected by dual SISH (Fig. 2). Of amplified cases, 27 cases $(81.8 \%)$ showed low-level amplification (ratio HER2/CEP17, 2 to $<5)$ and only six cases $(18.2 \%)$ showed a high ratio HER2/CEP17 (> 5). Polysomy of chromosome 17 was not found in any of the tissue samples.

The correlation between IHC and ISH in the surgical specimens is shown in Table 2. A concordance between IHC and ISH results was seen in 190/208 (157 IHC 0/1+ not amplified, 6 IHC 2+ amplified, and 27 IHC $3+$ amplified) with a rate of $91.3 \%$ ( $\kappa=$ 0.76). Six out of 23 IHC $2+$ cases $(26.1 \%)$ showed amplification and one out of $28 \mathrm{IHC} 3+$ cases $(3.6 \%)$, which showed signet ring cell carcinoma, was not-amplified.

\section{Correlation between clinicopathological findings and HER2 status}

The clinicopathological differences were shown in gastric carcinoma with or without HER2 protein expression or HER2 gene amplification. The HER2 protein expression and HER2 gene amplification rates were $43.6 \%$ (34/78) and 29.5\% (23/78) in intestinal type gastric carcinomas $(\mathrm{p}<.05)$. HER2 status was also related to good differentiation $(\mathrm{p}<.05)$. However, no significant correlation was found between HER2 status and other clinicopathological features including sex and age of the patients, depth of invasion, lymph node status, and lymphovascular invasion (Table 3).

\section{Intratumor heterogeneity of HER2}

HER2 showed phenotypic heterogeneity (IHC expression) and genotypic heterogeneity (gene amplification) (Fig. 3). Phenotypic heterogeneity was observed in 35 of $51 \mathrm{IHC}$ cases $(68.8 \%)$, in which 12 cases of $28 \mathrm{IHC} 3+$ cases $(42.9 \%)$ and all $23 \mathrm{IHC} 2+$ cases $(100 \%)$ demonstrated heterogeneity. Phenotypic heterogeneity of HER 2 expression was higher in the diffuse type than in the intestinal type (75\% vs $67.6 \%$ ). Genotypic heterogeneity was observed in 19 of 33 ISH cases (57.6\%). Genotypic heterogeneity was higher in diffuse-type than in intestinal-type cancers (66.7\% vs $52.2 \%)$. 


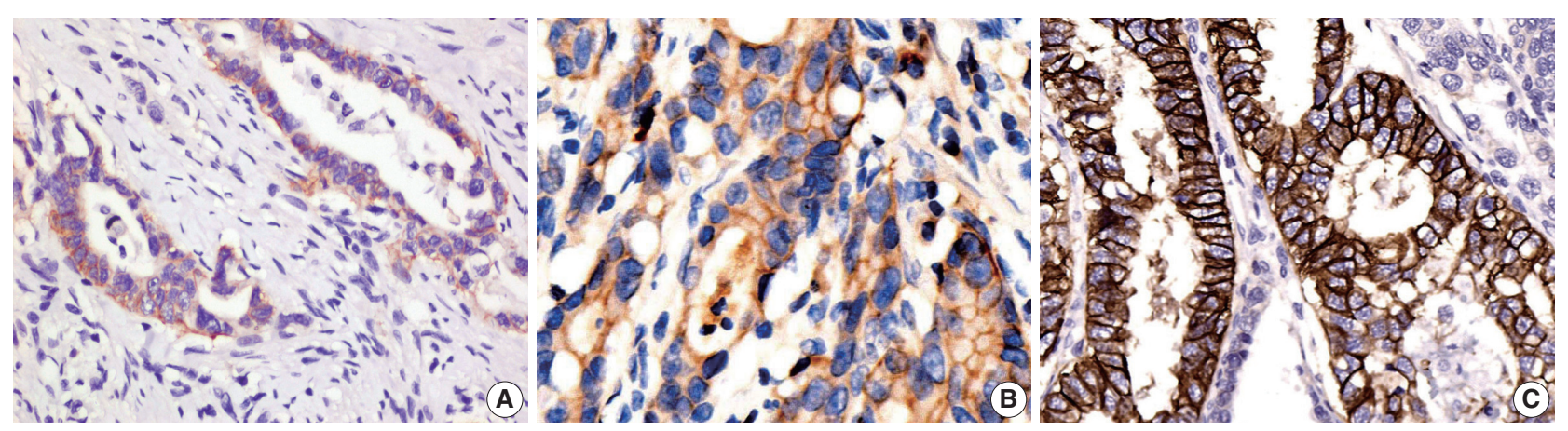

Fig. 1. The human epidermal growth factor receptor 2 (HER2) protein expression detected by immunohistochemistry (IHC). HER2 IHC scores of $1+(A), 2+(B)$, and $3+(C)$.
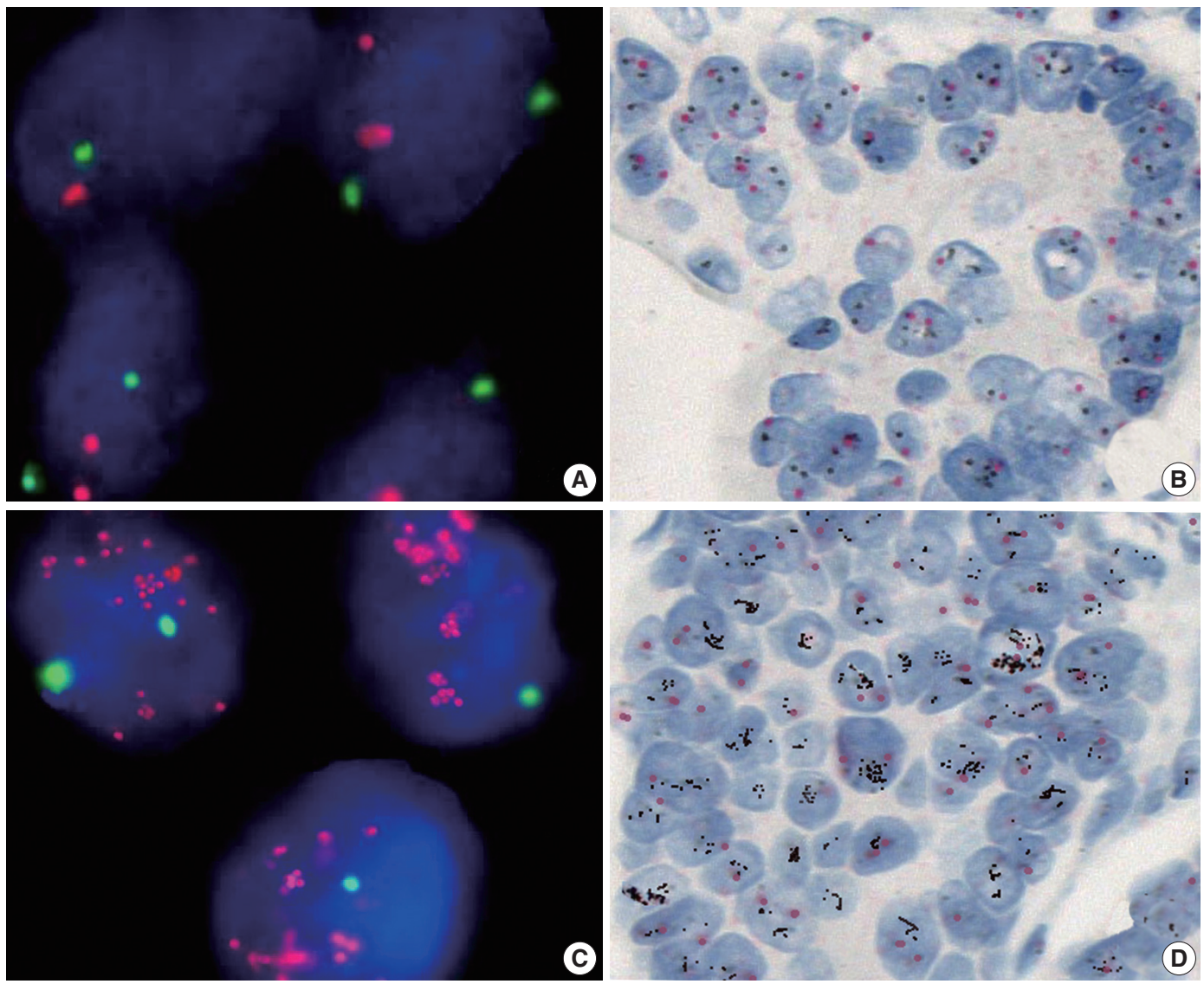

Fig. 2. The human epidermal growth factor receptor 2 (HER2) gene status detected by fluorescence in situ hybridization (FISH) and dual-color silver in situ hybridization (SISH). Representative photomicrographs of FISH (A, C) and dual-color SISH (B, D). Non-amplified cases detected by FISH (A) and dual-color SISH (B). Amplified cases detected by FISH (C) and dual-color SISH (D).

Table 2. Correlation between HER2 protein expression and HER2 gene amplification

\begin{tabular}{lccccc}
\hline HER2 & \multicolumn{5}{c}{ Immunohistochemistry for HER2 } \\
\cline { 2 - 6 } Dual ISH and FISH & $3+(\mathrm{n}=28)$ & $2+(\mathrm{n}=23)$ & $1+(\mathrm{n}=9)$ & $0(\mathrm{n}=148)$ & \\
\hline Amplified & 27 & 6 & 0 & 0 & Total \\
Not-amplified & 1 & 17 & 9 & 148 & 175 \\
\hline
\end{tabular}

HER2, human epidermal growth factor receptor 2; ISH, in situ hybridization; FISH, fluorescence in situ hybridization. 
Table 3. Correlation between clinicopathologic findings and HER2 status

\begin{tabular}{|c|c|c|c|c|}
\hline Clinicopathologic data & HER2 (IHC) positive & $p$-value & HER2 (ISH) amplification & $p$-value \\
\hline Sex & & NS & & NS \\
\hline Male & $37(26.6)$ & & 25 (18.0) & \\
\hline Female & 14 (20.3) & & $8(11.6)$ & \\
\hline Age (yr) & & NS & & NS \\
\hline$>60$ & $29(30.5)$ & & $18(18.9)$ & \\
\hline$\leq 60$ & $22(19.5)$ & & 15 (13.3) & \\
\hline Histologic type & & $<.05$ & & $<.05$ \\
\hline Intestinal type & 34 (43.6) & & $23(29.5)$ & \\
\hline Diffuse type & $12(11.0)$ & & $6(5.5)$ & \\
\hline Tumor differentiation & & $<.05$ & & $<.05$ \\
\hline Grade 1, 2 & $34(44.2)$ & & $22(28.6)$ & \\
\hline Grade 3, 4 & $17(13.0)$ & & $11(8.4)$ & \\
\hline Depth of invasion & & NS & & NS \\
\hline T1-2 & $13(27.1)$ & & $8(16.7)$ & \\
\hline T3-4 & $38(23.8)$ & & $25(15.6)$ & \\
\hline Lymph node metastasis & & NS & & NS \\
\hline Negative & $21(24.1)$ & & $13(14.9)$ & \\
\hline Positive & $30(24.8)$ & & $20(16.5)$ & \\
\hline Lymphovascular invasion & & NS & & NS \\
\hline Negative & $37(24.5)$ & & 23 (15.2) & \\
\hline Positive & $14(24.6)$ & & $10(17.5)$ & \\
\hline
\end{tabular}

Values are presented as number (\%).

HER2, human epidermal growth factor receptor 2; IHC, immunohistochemistry; ISH, in situ hybridization; NS, not significant.
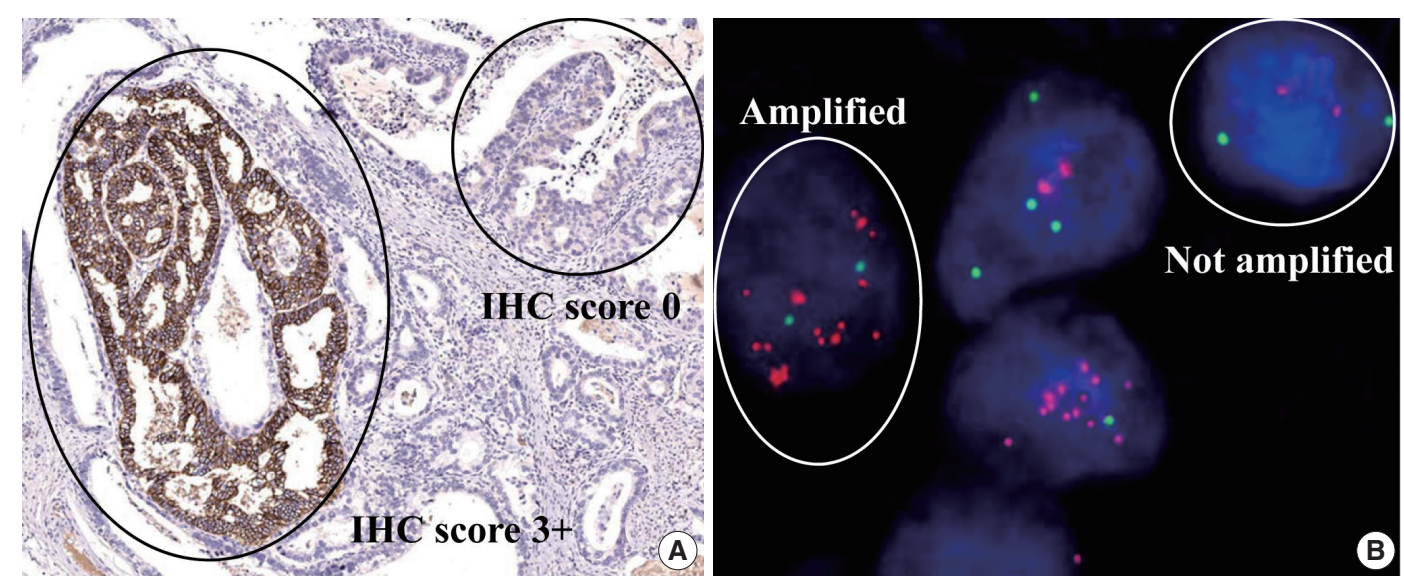

Fig. 3. Intratumoral heterogeneity of human epidermal growth factor receptor 2 (HER2). Heterogeneity of HER2 protein expression (A) and heterogeneity of HER2 gene amplification (B).

\section{DISCUSSION}

We investigated HER2 protein overexpression and gene amplification in 208 surgical tissue samples of gastric carcinoma. HER 2 protein positivity and HER2 gene amplification were observed in $24.5 \%$ and $15.9 \%$ of cases, respectively. While HER2 protein positivity in gastric carcinoma was reported from 11 to $24 \%,{ }^{6,8,11-13}$ HER2 gene amplification varied from $7 \%$ to $27 \% .^{7-10}$ This variation was attributed to an inconsistent HER2 IHC scoring system, different methodologies including antibody selec- tion and/or random selection of patients. Moreover, the interpretation of IHC or ISH can be influenced by subjective factors such as the prompt fixing time after removal, the volume of formalin, and the duration of fixation. In this study, all gastric specimens were fixed no later than 30 minutes after removal in $10 \%$ buffered formalin for $8-48$ hours.

In our study, we used the HER2 scoring system proposed by Hofmann et al., ${ }^{6}$ showing a high level of concordance (91.3\%) between IHC and ISH. A number of studies investigated the relationship between IHC and FISH in gastric cancer specimens 
and also reported a high level of concordance that varied from 86.9 to $93.5 \%$. ${ }^{3,6}$ Our study also showed high concordance between IHC and ISH. We did not compare the concordance between dual SISH and FISH in this study. However, some studies have shown a high level of concordance between dual SISH and FISH, which ranged from $94.5 \%$ to $98.3 \%$.

In this study, we recognized that one of 28 HER2 IHC 3+ cases showed non-amplification, and it was signet ring cell carcinoma. HER2 status may be found in this rare histologic type of gastric cancer with a very low rate of positivity. The HER2 protein expression in signet ring cell carcinoma may be misinterpreted due to strong nuclear/cytoplasmic staining, which was observed in the IHC staining method. Therefore, ISH confirmation should be considered in signet ring cell carcinoma.

The higher positive rates of HER2 in intestinal-type compared to other types of gastric carcinoma have been widely reported and our results were similar. ${ }^{8,11,13-16}$ It was also reported that HER2 overexpression is related to well-and-moderately differentiated gastric cancer but not with tumor stage or the age and sex of gastric cancer patients. ${ }^{13,17,18}$ On the other hand, the correlation of positive HER2 status with other clinicopathological factors, including age, sex, and TNM stage, remains controversial. It has been reported that a higher frequency of HER2 positivity is significantly correlated with older age, male gender, upper location of tumor, lymphatic or vascular invasion, higher $\mathrm{T}$ stage, lymph node metastasis, distant metastasis, and higher TNM stage. ${ }^{8,11,15,19-21}$ In our study, we demonstrated that there was no significant relationship between HER2 status and clinicopathological variables such as sex and age of the patients, depth of invasion, lymph node status and lymphovascular invasion.

Genotypic heterogeneity was defined by College of American Pathologists (CAP) in 2009 for breast cancers, in which the tumor showed at least $5 \%$ but fewer than $50 \%$ of nuclei with a HER2/ CEP17 ratio $\geq 2.0{ }^{22}$ The 2013 American Society of Clinical Oncology/CAP HER2 guideline update ${ }^{5}$ recommends using an IHC HER2 test to define areas of potential amplification. In gastric cancer, there are no guidelines for tumor heterogeneity assessment. Yang et al..$^{16}$ recognized $79.3 \%$ of cases with heterogeneous HER 2 protein expression and $44.0 \%$ of cases with HER2 genetic heterogeneity. Our results also showed that frequency of phenotypic heterogeneity was higher than that of genotypic heterogeneity $(68.8 \%$ vs $57.6 \%)$.

Kim et al..$^{11}$ found that heterogeneity was more prevalent in IHC $2+$ cases than IHC $3+$ cases $(90.9 \%$ vs $40.9 \%)$. Nishida $e t$ al..$^{23}$ also reported that phenotypic heterogeneity was recognized in $63.5 \%$ of IHC $2+$ cases, and in only $28.3 \%$ in IHC $3+$ cases.
Our results are consistent with results from these studies, in which phenotypic heterogeneity and genotypic heterogeneity showed a higher rate in IHC $2+$ cases than IHC $3+$ cases.

Lee et al. ${ }^{24}$ recognized that diffuse or mixed histological subtypes have a higher rate of heterogeneous HER2 expression. In our study, we also found that the diffuse type showed a higher rate of heterogeneity for HER2 expression or amplification than did the intestinal type. These results may be important for pathologists to consider when predicting HER2 heterogeneity in IHC and ISH results during routine histological examination.

In conclusion, our study demonstrates that HER2 protein expression and gene amplification were recognized in $24.5 \%$ and $15.9 \%$ of gastric cancer patients, respectively. Intestinal-type disease showed a higher rate of HER2 protein expression and gene amplification than diffuse type. HER 2 status also showed a significant relationship with well and moderately differentiated carcinomas. The ratio of phenotypic and genotypic heterogeneity of HER2 was high in gastric carcinoma and related to HER2 IHC $2+$ and diffuse histologic type.

\section{Conflicts of Interest}

No potential conflict of interest relevant to this article was reported.

\section{Acknowledgments}

We would like to give special thanks to Hoffmann La Roche Company and Roche Diagnostics Vietnam for HER2 IHC testing and dual-color SISH support and The Ho Chi Minh City Oncology Hospital, Vietnam, for HER2 FISH testing support.

\section{REFERENCES}

1. Ferlay J, Soerjomataram I, Dikshit R, et al. Cancer incidence and mortality worldwide: sources, methods and major patterns in GLOBOCAN 2012. Int J Cancer 2015; 136: E359-86.

2. Kuo CY, Chao Y, Li CP. Update on treatment of gastric cancer. J Chin Med Assoc 2014; 77: 345-53.

3. Bang YJ, Van Cutsem E, Feyereislova A, et al. Trastuzumab in combination with chemotherapy versus chemotherapy alone for treatment of HER2-positive advanced gastric or gastro-oesophageal junction cancer (ToGA): a phase 3, open-label, randomised controlled trial. Lancet 2010; 376: 687-97.

4. Zaczek A, Brandt B, Bielawski KP. The diverse signaling network of EGFR, HER2, HER3 and HER4 tyrosine kinase receptors and the consequences for therapeutic approaches. Histol Histopathol 2005; 
20: $1005-15$.

5. Wolff AC, Hammond ME, Hicks DG, et al. Recommendations for human epidermal growth factor receptor 2 testing in breast cancer: American Society of Clinical Oncology/College of American Pathologists clinical practice guideline update. J Clin Oncol 2013; 31: 39974013.

6. Hofmann M, Stoss O, Shi D, et al. Assessment of a HER2 scoring system for gastric cancer: results from a validation study. Histopathology 2008; 52: 797-805.

7. Takehana T, Kunitomo K, Kono K, et al. Status of c-erbB-2 in gastric adenocarcinoma: a comparative study of immunohistochemistry, fluorescence in situ hybridization and enzyme-linked immuno-sorbent assay. Int J Cancer 2002; 98: 833-7.

8. Yan SY, Hu Y, Fan JG, et al. Clinicopathologic significance of HER$2 /$ neu protein expression and gene amplification in gastric carcinoma. World J Gastroenterol 2011; 17: 1501-6.

9. Park YS, Hwang HS, Park HJ, et al. Comprehensive analysis of HER2 expression and gene amplification in gastric cancers using immunohistochemistry and in situ hybridization: which scoring system should we use? Hum Pathol 2012; 43: 413-22.

10. Yano T, Doi T, Ohtsu A, et al. Comparison of HER2 gene amplification assessed by fluorescence in situ hybridization and HER2 protein expression assessed by immunohistochemistry in gastric cancer. Oncol Rep 2006; 15: 65-71.

11. Kim KC, Koh YW, Chang HM, et al. Evaluation of HER2 protein expression in gastric carcinomas: comparative analysis of 1,414 cases of whole-tissue sections and 595 cases of tissue microarrays. Ann Surg Oncol 2011; 18: 2833-40.

12. Park DI, Yun JW, Park JH, et al. HER-2/neu amplification is an independent prognostic factor in gastric cancer. Dig Dis Sci 2006; 51: 1371-9.

13. Shan L, Ying J, Lu N. HER2 expression and relevant clinicopathological features in gastric and gastroesophageal junction adenocarcinoma in a Chinese population. Diagn Pathol 2013; 8: 76.

14. Barros-Silva JD, Leitao D, Afonso L, et al. Association of ERBB2 gene status with histopathological parameters and disease-specific survival in gastric carcinoma patients. Br J Cancer 2009; 100: 487-93.

15. Cho J, Jeong J, Sung J, et al. A large cohort of consecutive patients confirmed frequent HER2 positivity in gastric carcinomas with advanced stages. Ann Surg Oncol 2013; 20 Suppl 3: S477-84.

16. Yang J, Luo H, Li Y, et al. Intratumoral heterogeneity determines discordant results of diagnostic tests for human epidermal growth factor receptor (HER) 2 in gastric cancer specimens. Cell Biochem Biophys 2012; 62: 221-8.

17. Grabsch H, Sivakumar S, Gray S, Gabbert HE, Müller W. HER2 expression in gastric cancer: rare, heterogeneous and of no prognostic value: conclusions from 924 cases of two independent series. Cell Oncol 2010; 32: 57-65.

18. He C, Bian XY, Ni XZ, et al. Correlation of human epidermal growth factor receptor 2 expression with clinicopathological characteristics and prognosis in gastric cancer. World J Gastroenterol 2013; 19: 2171-8.

19. Moelans CB, Milne AN, Morsink FH, Offerhaus GJ, van Diest PJ. Low frequency of HER2 amplification and overexpression in early onset gastric cancer. Cell Oncol (Dordr) 2011; 34: 89-95.

20. Zhang XL, Yang YS, Xu DP, et al. Comparative study on overexpression of HER2/neu and HER3 in gastric cancer. World J Surg 2009; 33: 2112-8.

21. Yonemura Y, Ninomiya I, Yamaguchi A, et al. Evaluation of immunoreactivity for erbB-2 protein as a marker of poor short term prognosis in gastric cancer. Cancer Res 1991; 51: 1034-8.

22. Vance GH, Barry TS, Bloom KJ, et al. Genetic heterogeneity in HER2 testing in breast cancer: panel summary and guidelines. Arch Pathol Lab Med 2009; 133: 611-2.

23. Nishida $Y$, Kuwata T, Nitta $H$, et al. A novel gene-protein assay for evaluating HER2 status in gastric cancer: simultaneous analyses of HER2 protein overexpression and gene amplification reveal intratumoral heterogeneity. Gastric Cancer 2015; 18: 458-66.

24. Lee S, de Boer WB, Fermoyle S, Platten M, Kumarasinghe MP. Human epidermal growth factor receptor 2 testing in gastric carcinoma: issues related to heterogeneity in biopsies and resections. Histopathology 2011; 59: 832-40. 